\title{
Cognitive control signals in posterior cingulate cortex
}

\author{
Benjamin Y. Hayden ${ }^{1,2,3 *}$, David V. Smith ${ }^{2,3,4}$ and Michael L. Platt ${ }^{1,2,3,5}$ \\ 1 Department of Neurobiology, Duke University School of Medicine, Durham, NC, USA \\ 2 Center for Neuroeconomic Studies, Duke University School of Medicine, Durham, NC, USA \\ ${ }^{3}$ Center for Cognitive Neuroscience, Duke University, Durham, NC, USA \\ ${ }^{4}$ Department of Psychology, Duke University, Durham, NC, USA \\ ${ }^{5}$ Department of Evolutionary Anthropology, Duke University, Durham, NC, USA
}

Edited by:

Sven Bestmann, University College

London, UK

\section{Reviewed by:}

Bruno B. Averbeck, National Institute of

Mental Health, USA

Veit Stuphorn, Johns Hopkins

University, USA

${ }^{*}$ Correspondence:

Benjamin Y. Hayden, Department of Neurobiology, Duke University Medical School, Durham, NC 27710, USA. e-mail: hayden@neuro.duke.edu

\begin{abstract}
Efficiently shifting between tasks is a central function of cognitive control. The role of the default network - a constellation of areas with high baseline activity that declines during task performance - in cognitive control remains poorly understood. We hypothesized that task switching demands cognitive control to shift the balance of processing toward the external world, and therefore predicted that switching between the two tasks would require suppression of activity of neurons within the posterior cingulate cortex (CGp). To test this idea, we recorded the activity of single neurons in CGp, a central node in the default network, in monkeys performing two interleaved tasks. As predicted, we found that basal levels of neuronal activity were reduced following a switch from one task to another and gradually returned to pre-switch baseline on subsequent trials. We failed to observe these effects in lateral intraparietal cortex, part of the dorsal fronto-parietal cortical attention network directly connected to CGp. These findings indicate that suppression of neuronal activity in CGp facilitates cognitive control, and suggest that activity in the default network reflects processes that directly compete with control processes elsewhere in the brain.
\end{abstract}

Keywords: default network, executive function, lateral intraparietal cortex, task-switching

\section{INTRODUCTION}

The ability to switch efficiently between different tasks in the behavioral repertoire is a critical component of cognitive flexibility. A wealth of studies has established that the fronto-parietal network plays a critical role in the cognitive control processes required for efficient task switching (Dove et al., 2000; Sohn et al., 2000; Nakahara et al., 2002; Rushworth et al., 2002, 2003; Aron et al., 2004; Brass et al., 2005; Crone et al., 2006; Hyafil et al., 2009). By contrast, we know almost nothing about the role of the complementary default network in task switching and cognitive control. This network, which includes the posterior cingulate cortex (CGp), the ventromedial prefrontal cortex, the temporal-parietal junction, and other areas, shows high baseline activity during periods of rest that is suppressed during task engagement (Gusnard and Raichle, 2001; Raichle et al., 2001; Raichle and Mintun, 2006; Buckner et al., 2008; Hayden et al., 2009).

Variations in default network activity have behavioral consequences. Elevated hemodynamic activity in the default network predicts momentary lapses in attention and failures to perceive and encode external stimuli (Daselaar et al., 2004; Weissman et al., 2006; Boly et al., 2007). Enhanced tonic activity of neurons in macaque CGp predicts slower reaction times and higher error rates (Hayden et al., 2009). Because monkeys appear to have a default network homologous with that of humans (Vincent et al., 2006; Hayden et al., 2009), these neuronal effects likely extend to humans as well. These observations suggest that neural activity in CGp, and perhaps the default network more generally, reflects processes that compete with cognitive control processes elsewhere in the brain.
To examine this idea more closely, we re-analyzed a previously acquired dataset that was designed to characterize default network activity at the single neuron level (Hayden et al., 2009). Our experiment interleaved two distinct instrumental tasks - a cued gaze-shift task and a delayed gaze-shift task - as well as a cued rest period, which we did not examine further here. Switching from one task to another is a well-studied executive control problem (Monsell, 2003). People and monkeys performing simple laboratory experiments typically show slower reaction times when they switch from one task to another, indicative of a cognitive cost (Rogers and Monsell, 1995; Rushworth et al., 2003; Altmann, 2004; Stoet and Snyder, 2009). Task-switching costs occur in many behavioral contexts, are observed with even simple psychophysical tasks (Logan and Bundesen, 2003; Altmann, 2004), and are not eliminated by prior cueing (Monsell, 2003) or by extensive practice (Stoet and Snyder, 2007). Several brain regions, especially in the fronto-parietal network and closely connected regions, have been clearly linked to the cognitive control processes engaged by task switching (Dove et al., 2000; Sohn et al., 2000; Nakahara et al., 2002; Rushworth et al., 2002, 2003; Aron et al., 2004; Brass et al., 2005; Crone et al., 2006; Hyafil et al., 2009).

Based on these observations, we hypothesized that task switching demands cognitive control to shift the balance of processing toward the external world, and therefore predicted that switching between the two tasks would require suppression of activity of neurons within the CGp. Consistent with our hypothesis, we found that firing rates of CGp neurons were lowest following task switches, and gradually increased on subsequent trials of the same type. We observed no such effects in lateral intraparietal cortex (LIP), a brain 
region that is directly connected to CGp, but outside the default network. These findings indicate that suppression of neuronal activity in CGp contributes to cognitive control, and suggest that activity in the default network reflects processes that directly compete with control processes elsewhere in the brain.

\section{MATERIALS AND METHODS}

The data analyzed in this paper were originally collected for another study and published (Hayden et al., 2009) but the analyses presented here are new. Standard surgical and behavioral procedures were used (for details, see Dean et al., 2004). All procedures were approved by the Duke University Institutional Animal Care and Use Committee and were designed and conducted in compliance with the Public Health Service's Guide for the Care and Use of Animals.

Two male rhesus macaques (Macaca mulatta) served as subjects. A small head-restraint prosthesis was implanted in both animals using standard surgical techniques. Four weeks later, animals were habituated to laboratory conditions and trained to perform tasks for liquid reward. A second surgical procedure was then performed to place a stainless steel chamber (Crist Instruments) over CGp (in one monkey) and over LIP (in the other monkey). In both cases, one recording location was approached through a standard vertical recording grid (CGp for monkey $\mathrm{N}$ and LIP for monkey D), whereas the other area was approached using an angled grid (LIP for monkey $\mathrm{N}$ and CGp for monkey D). The posterior cingulate chamber (subject $\mathrm{N}$ ) was positioned at the intersection of the midsagittal plane and the interaural plane. The LIP chamber (subject D) was placed $3 \mathrm{~mm}$ caudal and $12 \mathrm{~mm}$ lateral to the intersection of the midsagittal and interaural planes. Animals received analgesics and antibiotics after all surgeries.

Throughout both behavioral and physiological recording sessions, the chamber was kept sterile with regular antibiotic washes and sealed with sterile caps. Although we recorded in both the cingulate gyrus and along the sulcus, we have chosen to use the term CGp for consistency with previous papers. In any case, we found no functional distinction between neurons recorded along the sulcus or gyrus, nor did we find any gradient of functions at different locations, either dorsoventrally or rostrocaudally.

\section{BEHAVIORAL PROCEDURES}

Monkeys were placed on controlled access to fluid outside of experimental sessions. Horizontal and vertical eye positions were sampled at $1000 \mathrm{~Hz}$ by an infrared eye-monitoring camera system (SR Research, Osgoode, ON, USA). Stimuli were controlled by a computer running Matlab (Mathworks, Natick, MA, USA) with Psychtoolbox (Brainard, 1997) and Eyelink Toolbox (Cornelissen et al., 2002). Visual stimuli were small colored squares on a computer monitor placed directly in front of the animal and centered on his eyes. A standard solenoid valve controlled the duration of juice delivery. Reward volume was $0.2 \mathrm{~mL}$ in all cases.

Monkeys sat in front of a computer monitor in a darkened room. Trials were one of three types (for most neurons) or four types (for a subset of 30 CGp neurons). The first three types were the attentive task, working memory task, and the no-task condition. The fourth type was the cued rest condition. The three (or four) trial types were interspersed randomly so that tasks sometimes repeated and sometimes switched between trials. Trials were not otherwise blocked. Task details are illustrated in Figure 1A.

In the attentive and working memory tasks, the trial began with the appearance of a small yellow fixation square ( 16 pixels, $\sim 0.5^{\circ}$ of visual angle). This stimulus lasted for $200 \mathrm{~ms}$ and then changed color to indicate which task or condition would be performed. A red cue signaled a memory task, a green cue signaled the saccade task, and the cue remained yellow for cued rest. There was no requirement that the monkey had to acquire fixation immediately, although monkeys typically acquired fixation within 2 s. Once fixation was acquired, the colored square immediately shrunk to indicate that the trial would begin. The resulting target remained on for $4 \mathrm{~s}$ (monkey N) or $3 \mathrm{~s}$ (monkey D) and was then extinguished, cuing the animal to shift gaze.

In the working memory task, an eccentric cue appeared after $2 \mathrm{~s}$ of fixation (monkey N) or $1 \mathrm{~s}$ (monkey D). The memory cue remained illuminated for $1 \mathrm{~s}$ and then disappeared. In the attentive task, an eccentric cue appeared at the end of the delay and the monkey was rewarded for shifting gaze to it. In the working memory
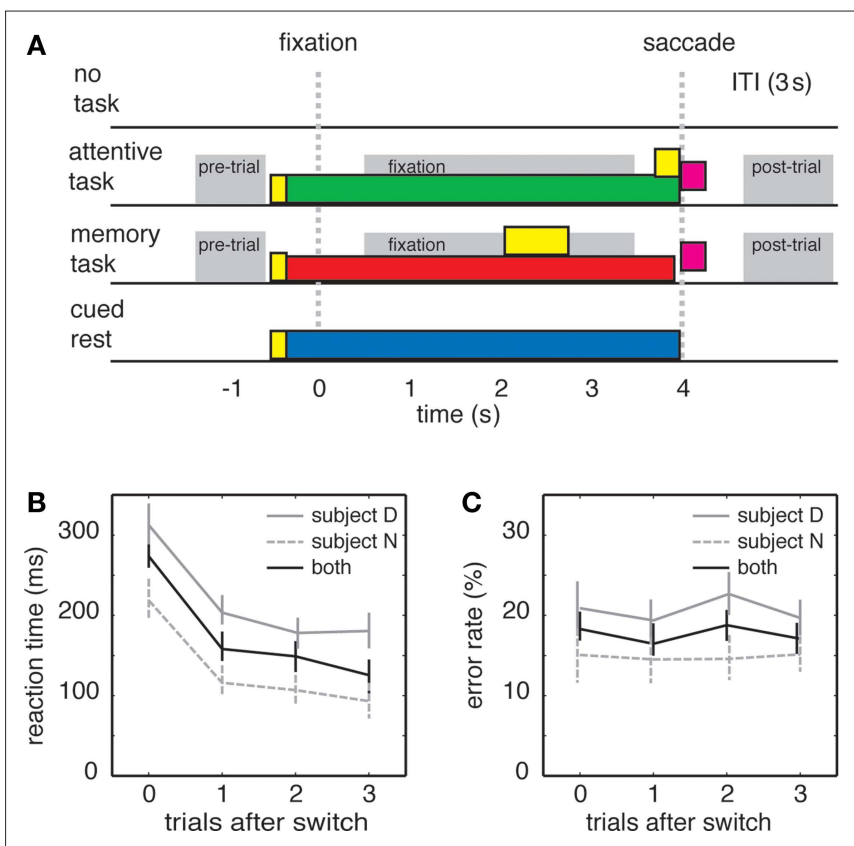

FIGURE 1 |Task switching influences behavioral performance in rhesus macaques. (A) Schematic of the four tasks; time zero indicates time at which fixation was acquired. In three of the tasks, a yellow small central cue was illuminated (yellow bar). After $200 \mathrm{~ms}$, the cue changed color (green for attentive, red for memory, yellow for cued rest). After shifting gaze toward the spot, the monkey maintained fixation for $4 \mathrm{~s}$ (monkey $\mathrm{N}$, shown) or $3 \mathrm{~s}$ (monkey D). In the working memory task, an eccentric cue appeared during the delay and remained illuminated for $1 \mathrm{~s}$ (yellow bar). Following the fixation period, the cue was extinguished and, in the attentive task, an eccentric target appeared at 1 of 36 locations from a grid surrounding the central cue (yellow square). Monkeys received a reward for shifting gaze to eccentric target or the remembered location (purple squares). Gray bars indicate epochs used for analysis. (B) Plot of reaction times for monkey N (gray-dashed), monkey D (gray), and average for both subjects (black). Bars indicate standard error. (C) Error rates of monkey N (gray-dashed), monkey D (gray), and average for both subjects (black). Bars indicate one standard error. 
task, no eccentric cue appeared, and the monkey had to shift gaze to the remembered location. In both tasks, target position was chosen from a $6 \times 6$ grid of locations on the monitor ( 36 possible locations spanning $\sim 14^{\circ}$ of visual angle both horizontally and vertically). A fluid reward was given following successful completion of either task. In the cued rest condition, no other stimuli appeared and no reward was given. In the no-task condition, no cue appeared, no fixation was required, and no reward was given. Inter-trial intervals (ITIs) were fixed at $3 \mathrm{~s}$ in all cases. The task used on each trial was selected at random from the menu of tasks, and thus task switches were unpredictable, and were determined post hoc.

\section{MICROELECTRODE RECORDING TECHNIQUES}

Single electrodes (Frederick Haer Co, Bowdoin, ME, USA) were lowered by hydraulic microdrive (Kopf) until the waveform of a single (1-4) neuron(s) was isolated. Individual action potentials were identified by their unique waveforms and isolated on a Plexon system (Plexon Inc, Dallas, TX, USA). All sorting was done online; no post-recording selection was performed. Neurons were selected for recording on the basis of the quality of isolation only. In all cases, neurons were considered isolated only if their waveforms were distinct from those of other neurons and background hash. Ultrasound images taken in the sagittal plane confirmed that the CGp recordings were made in areas 23 and 31 in the cingulate gyrus and ventral bank of the cingulate sulcus. This method has been used to confirm the positioning of CGp in several earlier studies (e.g., McCoy et al., 2003; McCoy and Platt, 2005; Hayden et al., 2008). For details on this method, see (Glimcher et al., 2001). We found no relationship between recording position and functional properties of neurons within CGp or LIP.

Peri-stimulus time histograms (PSTHs), plots of the average firing of neurons over time, were constructed by smoothing raw spike times (100 ms boxcar). For several analyses, average firing rates were calculated for each trial in three epochs (e.g., Figure 2B). The pretrial epoch began $1.5 \mathrm{~s}$ before fixation was acquired and lasted $1 \mathrm{~s}$. The fixation epoch began $500 \mathrm{~ms}$ after fixation was acquired and lasted $3 \mathrm{~s}$ ( $2 \mathrm{~s}$ in monkey D). The post-trial epoch began $500 \mathrm{~ms}$ after the reward and lasted for $1 \mathrm{~s}$. In analyses of responses in the post-trial epoch, we used a bin beginning at fixation and ending $2 \mathrm{~s}$ after reward ( $\sim 6 \mathrm{~s}$ in monkey $\mathrm{N}$ and $\sim 5 \mathrm{~s}$ in monkey D.).

\section{RESULTS}

\section{TASK SWITCHING SLOWS REACTION TIMES IN MONKEYS}

We randomly interleaved two tasks, a cued gaze-shift task (attentive) and a delayed gaze-shift (working memory) task (Figure 1A). We also interleaved a "no-task" condition, consisting simply of a 4-s delay. For a minority of neurons ( $n=30$ cells), we also interleaved a "cued rest" condition, consisting of a cue indicating that no task would be performed for $4 \mathrm{~s}$. The conditions occurred with equal probability and were randomly selected on a trial-by-trial basis.
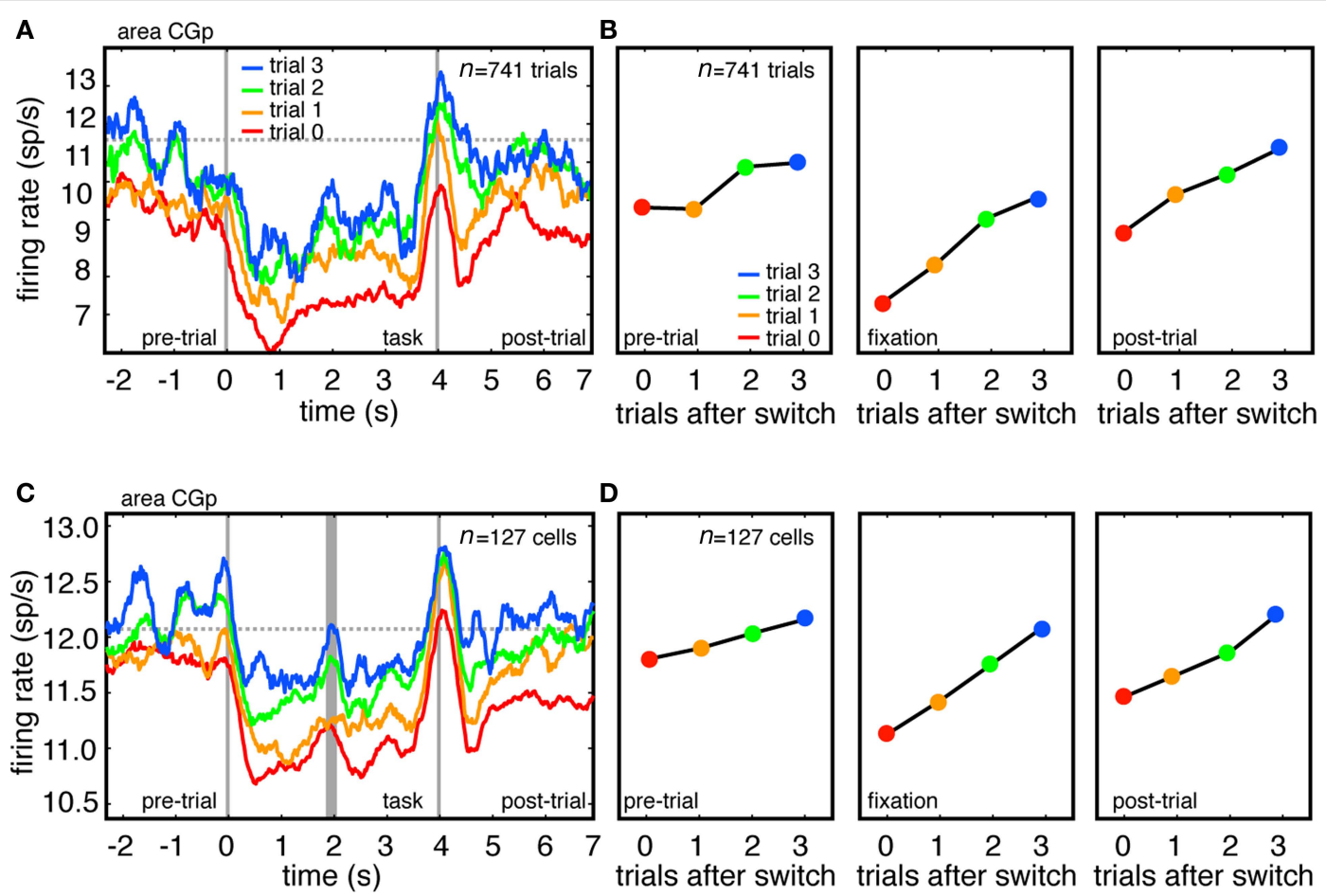

FIGURE 2 | Firing rates of CGp neurons track task switches. (A) Responses of example CGp neuron immediately following switch (trial 0), and on first, second, and third trials following switch. Responses are aligned to the time at which the computer detected that fixation had been successfully acquired (time 0 ).

Horizontal dashed line indicates average baseline firing during no-task condition, and provides a rough baseline of undriven activity. (B) Plot of average firing rate during each of three task epochs (pre-trial, fixation, post-trial). (C) Responses of population of CGp neurons immediately after switch, and on first, second, and third trial following switch. Because tasks were different lengths in monkeys $\mathrm{N}$ $(4 \mathrm{~s})$ and $D(3 \mathrm{~s})$, responses are separately aligned to trial onset (time $=0$ ) and trial offset (time $=4$ ), separated by thick gray line. (D) Plot of average firing rate during each of three task epochs (pre-trial, fixation, post-trial across population). 
We sorted tasks post hoc into switches and repetitions. Because the no-task and cued rest conditions did not require any overt behavior, we focused exclusively on neural activity during working memory and attentive tasks in our analyses, and excluded trials of the same type separated by a no task or cued rest trial. Because trial type was selected at random on each trial, the history of previous trials provided no information about the likelihood of an upcoming switch.

Reaction times are typically used as an index of task-switching costs. We calculated saccade reaction times one, two, and three trials after switches (Figure 1B). We found that reaction times were significantly increased after switches, and fell for subsequent trials ( $\beta=-45.4 \mathrm{~ms} /$ trial, $p<0.001$, linear regression). In this and subsequent tests, we regressed either reaction time or firing rate (see below) against number of trials after switch $(1,2,3$, or 4$)$ with data from several 100 individual trials (excluding from analysis trials of the same type separated by a no-task condition). (For this reaction time analysis, we combined data across all behavioral sessions, $n=61,087$ trials). We observed significant effects in both monkeys individually ( $\beta=-41.0 \mathrm{~ms} /$ trial and $\beta=-48.8 \mathrm{~ms} /$ trial for the two monkeys individually, $p<0.001$ in both cases, linear regression). These results demonstrate that the specific tasks and timing we used in our experiments elicited reliable behavioral task-switching costs. We observed no statistical relationship between the type of task switching (attentive to memory or memory to attentive) and reaction time (one factor ANOVA, reaction time against switch type, $p=0.45$ ).

Error rates are sometimes used as an index of task switching as well, although this measure has proven to be inconsistent (Monsell, 2003). Indeed, we found no effects of task switching on error rates in our experiment (Figure 1C). We defined errors as trials on which monkeys failed to hold fixation for the specified duration of the task ( 4 s for monkey N, 3 s for monkey D). In the working memory task, monkeys occasionally made errors by shifting gaze to the wrong spatial location. However, these trials were so infrequent $(<5 \%$ of error trials, $<1 \%$ of all trials) that we did not have enough data to perform meaningful statistical analyses. We observed no relationship between error rate and trials after switch in either monkey (monkey D: $\beta=-0.12, p=0.33$; monkey $\mathrm{N}: \beta=0.05, p=0.61$; linear regressions). We therefore did not analyze errors further. The lack of an error effect may derive from the simplicity of the tasks or a speed-accuracy tradeoff.

\section{SWITCHING TASKS SUPPRESSES FIRING RATES OF CGp NEURONS}

We recorded responses of 127 neurons in the CGp of two monkeys performing this task (83 in monkey $\mathrm{N}$ and 44 in monkey $\mathrm{D}$ ). We next examined the relationship between task switching and firing rates. Figure $2 \mathrm{~A}$ shows the average activity of a single CGp neuron on the switching trial itself (red), one trial after a switch (orange), two trials after a switch (green), and three trials after a switch (blue). (Because errors may promote enhanced firing rates, trials following error trials were excluded from these analyses). Because trials were randomly interleaved and organized into switches and repeats post hoc, we did not have enough data to analyze trials beyond three after a switch. The second vertical gray bar indicates the time of the command to initiate a gaze shift, and corresponds closely to the time of both the eye movement and the time of the reward (movement and reward were time-locked by the design of the task).
We next calculated the average firing rates during each of three epochs (Figure 2B). The pre-trial epoch was a $1 \mathrm{~s}$ epoch beginning $1.5 \mathrm{~s}$ before fixation was acquired and lasting $1 \mathrm{~s}$. The fixation epoch began $500 \mathrm{~ms}$ after fixation was acquired and lasted $3 \mathrm{~s}(2 \mathrm{~s}$ in monkey D). The post-trial epoch began $500 \mathrm{~ms}$ after the reward and lasted for $1 \mathrm{~s}$. Note that firing rate modulations occurring before cue onset are to be expected given the design of our analyses. The pre-cue neural activity for the four-trial-post-switch line (blue line) reflects all trials with three of the same task type in a row, while the pre-cue neural activity for the three-trial-post-switch line (green line) reflects only trials with two of the same type in a row, and so on. These long-lasting modulations that persist across the delay between trials seem to be the rule rather than the exception in CGp, a phenomenon we have discussed in detail previously (Hayden et al., 2008).

Tonic firing rates for this example neuron, during all epochs, were lowest following a task switch, and increased on subsequent trials (Figure 2B, $\beta=0.46 \mathrm{sp} / \mathrm{s}$ pre-trial, $\beta=0.94 \mathrm{sp} / \mathrm{s}$ during the trial, and $\beta=0.67 \mathrm{sp} / \mathrm{s}$ post-trial, $p<0.001$, in all cases, linear regression). We observed a weak effect of task type on firing rates, with memory trials showing a reduced neuronal response during the final second of the fixation, as reported previously (Hayden et al., 2009). We observed no statistical interaction between task type (attentive or memory) and trials since task switch on firing rates (two-way ANOVA, task switch type by switch number, $p=0.60$ ).

We next repeated these analyses for all 127 CGp neurons in our sample. We observed the same pattern in the average neuronal activity of all neurons in our sample as we observed in the example neuron (Figures 2C,D $\beta=0.48 \mathrm{sp} / \mathrm{s}$ pre-trial, $\beta=0.99 \mathrm{sp} / \mathrm{s}$ during the trial, and $\beta=0.89 \mathrm{sp} / \mathrm{s}$ post-trial, $p<0.001$ in all cases, linear regression). Note that these PSTHs include data from all neurons we recorded, with no pre-selection based on functional properties. For the population PSTH, data were not normalized, but instead reflect raw average firing rates. A significant relationship between firing rate and trial number after switch during the fixation epoch was observed in $41 \%(n=52 / 127,36 / 83$ in monkey $\mathrm{N}, 16 / 44$ in monkey D) of neurons ( $p<0.05$, linear regression). This relationship was observed during the pre-trial epoch in $34 \%(n=43 / 127$, $29 / 83$ in monkey $N, 14 / 44$ in monkey D) of neurons and during the post-trial epoch in $38 \%(n=48 / 127,32 / 83$ in monkey $\mathrm{N}, 16 / 44$ in monkey D) of neurons.

These proportions themselves are significant (with an alpha of $p=0.05,5 \%$ of neurons -8.3 - would be expected to show a significant effect in each epoch). To provide additional confirmation for this claim, we performed a randomization test in which we randomly assigned "trials after switch" to each trial, and recalculated the relationship between firing rate and trials after switch. We then repeated this randomization process 10,000 times for each neuron in the set of 127 neurons, and estimated the average number of significant neurons observed in each randomization. As expected the mean and modal number of significant neurons was 8 .

Of the neurons significantly modulated during the fixation epoch, the majority $(85 \%, n=44 / 52,31$ in monkey N, 13 in monkey D) showed a positive correlation with trial since a task switch. The remaining eight neurons showed a significant negative correlation, while the remaining 75 showed no significant effects. A visual inspection of the response profile of these neurons with 
no significant effects confirmed that firing rates did not change with trial number after a switch. The effect of trial number since switch on firing rates was largely linear. The addition of a quadratic term in the regression improved the fit for only $19.2 \%$ of neurons ( $n=10 / 52$ significantly modulated neurons, six in monkey $\mathrm{D}$, four in monkey D). Despite this linearity, the effect of trials since switch on reaction time was highly non-linear: the effect of the first trial was much stronger than the effect of subsequent trials, as in other studies. These results indicate that firing rates in CGp do not strictly track reaction time, but instead appear to index cognitive processes that only partially predict reaction times.

We previously showed that different tasks evoke different effects on tonic firing rates, possibly because they elicit different degrees of exteroceptive engagement (Hayden et al., 2009). It is therefore possible that different types of task switches may elicit distinct neural effects. However, we observed no statistical relationship between type of task switch and firing rates (two-way ANOVA, task switch type by switch number, $p=0.21$ ). Furthermore, although we found weak and broad tuning for saccade direction, consistent with previous results (Dean et al., 2004; Hayden et al., 2008), we observed no interactions between these spatial effects and task-switching variables; therefore we have averaged over all spatial locations in the results described here (multi-factor ANOVA, factors of $X$ and $Y$ position - six levels each - and switching status on firing rate, $p=0.61)$.

\section{SWITCHING TASKS DOES NOT MODULATE FIRING RATES OF LIP NEURONS}

We also recorded the activity of 55 single neurons in area LIP in the same two monkeys in different recording sessions. LIP is a parietal region that is directly connected with CGp (Kobayashi and Amaral, 2003), but is not part of the default network. Instead, this area is a node in the complementary dorsal fronto-parietal attentional control network (Corbetta et al., 2002; Smith et al., 2010) and activity in this network has been shown, in neuroimaging studies, to be anti-correlated with activity in the default network (Fox et al., 2005; Vincent et al., 2007).

The responses of both an example LIP neuron (Figures 3A,B) and the population of LIP neurons (three neurons with insufficient number of trials were excluded, Figures 3C,D) showed clear phasic responses around the time of saccades that begin and end the trials. As above, these data include all recorded LIP neurons, with no pre-selection based on response properties, and are not normalized, but instead reflect raw firing rates. For both the example LIP neuron and the LIP population, we observed no relationship between neuronal activity and trial number after the switch ( $p=0.21$ and $p=0.90$ for the two monkeys respectively, linear regression). We observed significant relationships between firing rate and trial number in only $9 \%$ of LIP neurons $(n=5 / 55, p<0.05$, linear regression). Firing rates were positively correlated with trials after switch in $4 / 5$ of these. These results imply that the association
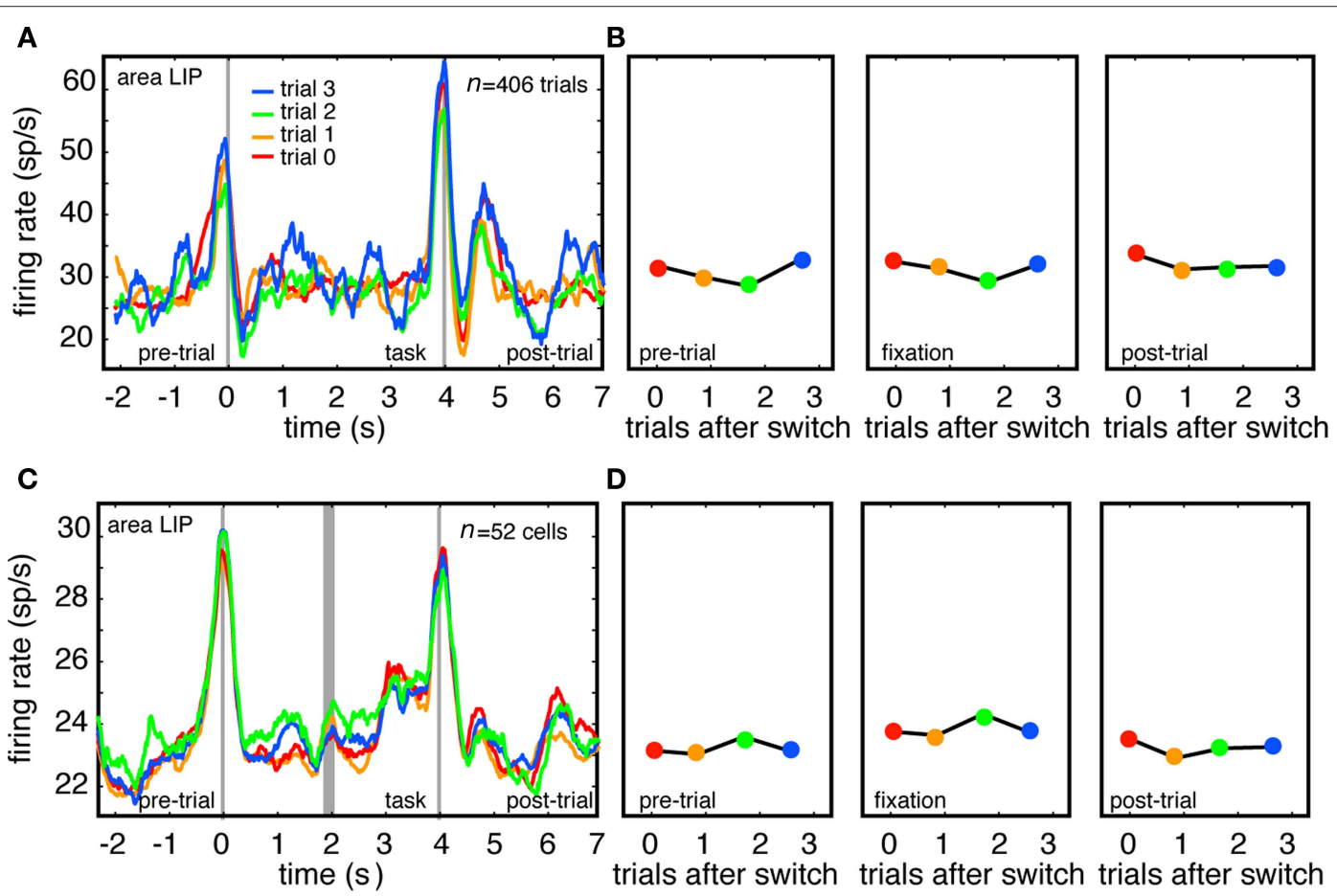

FIGURE 3 | Firing rates of LIP neurons do not track task switches.

(A) Responses of example LIP neuron immediately following switch (trial 0), and on first, second, and third trials following switch. Responses are aligned to the time at which the computer detected that fixation had been successfully acquired (time 0). (B) Plot of average firing rate during each of three task epochs (pre-trial, fixation, post-trial). (C) Responses of population of LIP neurons immediately after switch, and on first, second, and third trial following switch. Because tasks were different lengths in monkeys N (4 s) and $D(3 \mathrm{~s})$, responses are separately aligned to trial onset (time $=0$ ) and trial offset (time $=4$ ), separated by thick gray line. (D) Plot of average firing rate during each of three task epochs (pre-trial, fixation, post-trial across population. 
between firing rates and switching is not observed throughout the cortex, and are consistent with the idea that this pattern is limited to the default network.

\section{DEPTH OF FIRING RATE SUPPRESSION IN CGp, BUT NOT LIP, PREDICTS REACTION TIMES}

We report here that CGp firing rates are reduced following successful task switches (Figure 1B), whereas we have previously shown that variation in tonic firing rates of CGp neurons predicts variability in reaction times within a single task (Hayden et al., 2009). Together, these results suggest that reduced CGp activity indexes control processes that dictate switching efficiency, and that such processes are most engaged on efficient (i.e., rapid) switch trials, less engaged on inefficient (i.e., slow) switch trials, and least engaged on non-switch trials.

To examine this possibility, we analyzed the relationship between firing rates and reaction times on switch trials and non-switch trials separately. We defined short-RT trials as those where reaction time was less than the median RT for the session, and long-RT trials as those where reaction time was more than the median RT for the session. We found that neuronal activity was lower on short-RT switching trials than on long-RT switching trials. These effects were significant for both the example CGp neuron and for the CGp population (Figure 4, $t=3.26$ for the neuron, and $t=3.8$ for the population, $p<0.001$ in both cases, Student's $t$-test). Using this same test on each CGp neuron in the sample, we observed a significant relationship between firing rate and reaction time after switches in $41 \%$ of neurons following a task switch $(n=52 / 127,38$ in monkey $\mathrm{N}$ and 14 in monkey $\mathrm{D}$, linear regression, $p<0.05)$. The majority of these $(79 \%, n=41 / 52,31$ in monkey $\mathrm{N}, 10$ in monkey $\mathrm{D})$ showed a positive correlation between firing rate and RT. We also found that neurons were less active on short-RT no-switch trials than on long-RT no-switch trials, consistent with our previous results ( $t=4.2$ for the population, $p<0.001$, Hayden et al., 2009). Although we observed main effects of both trials after switch and reaction times within condition, we found no statistical interaction between these variables at the population level (two-way ANOVA, $p=0.36)$.

We did not observe analogous correlations between firing rates and reaction times in LIP. In the example LIP neuron (Figure 3C) and the LIP population (Figure 3D), no correlation between firing rates and reaction time were observed ( $p=0.54$, correlation test). These effects are consistent with our previous results showing that tonic firing rates are positively correlated with reaction times in CGp neurons, but are uncorrelated with firing rates of LIP neurons (Hayden et al., 2009).

\section{DISCUSSION}

We found that tonic firing rates of CGp neurons in monkeys are reduced following task switches. These reductions in firing rate co-occurred with increases in reaction time thought to index control processes associated with disengaging from one cognitive task set and entering a new one. These results implicate variations in neuronal activity within the default network in resetting cognitive state to achieve behavioral goals.

One parsimonious explanation for our data is that control processes associated with efficient task switching have an antagonistic relationship with activity in CGp, and that these processes are most robust, and CGp firing rates correspondingly weakest, on trials where resetting is most efficient (i.e., short-RT switch trials). Such
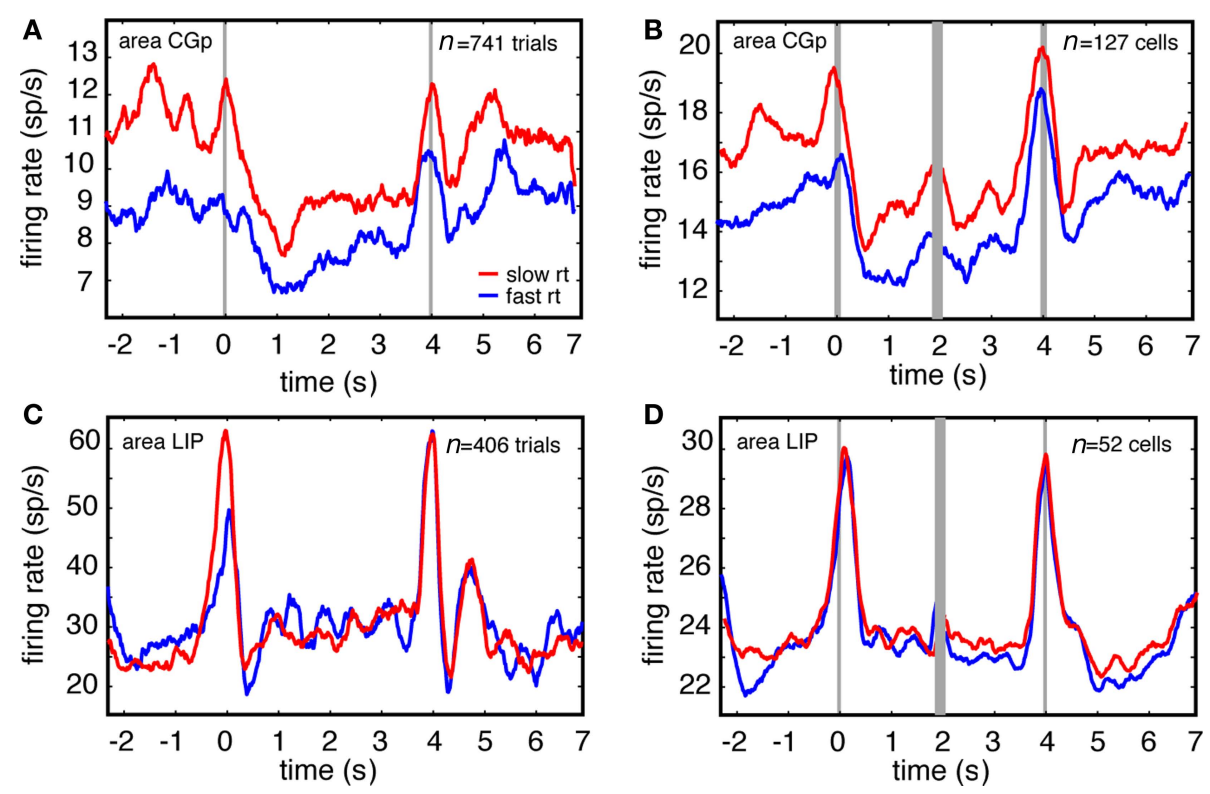

FIGURE 4 | Firing rates of single CGp neurons, but not LIP neurons, predict efficiency of switching, as indexed by reaction time. (A) Responses of example CGp neuron on fast (trials faster than median RT, blue) and slow (trials slower than median $\mathrm{RT}$, red) reaction time trials immediately following task switches. Responses are aligned to trial onset $(t=0)$. (B) Responses of population

of CGp neurons on fast and slow reaction time trials immediately following switches. Responses are separately aligned to trial onset (time $=0$ ) and trial offset (time =4). (C) Responses of example LIP neuron on fast and slow reaction time trials immediately following task switches. (D) Responses of population of LIP neurons on fast and slow reaction time trials immediately following task switches. 
processes may include retrieval and encoding of information in long-term memory (Daselaar et al., 2004), self-directed cognition (Gusnard et al., 2001; McKiernan et al., 2003) and environmental monitoring (Mason et al., 2007). When the processes that promote efficient resetting are less robust, and CGp firing rates are correspondingly higher, RTs on switch trials are slower. Resetting processes become systematically weaker on later non-switch trials as demand for cognitive control is reduced. Within this context, deeper firing rate suppression in CGp facilitates task switching.

On the surface, these results appear to diverge with our previous results showing that increased firing rates in CGp correlate with increased reaction times for performing a single task (Hayden et al., 2009). However, when considered together, our findings suggest that CGp firing rates do not signal reaction time per se, but instead decrease with cognitive processes that are marshaled both during efficient task switching and during efficient task performance. Collectively, therefore, these results endorse an emerging idea that reductions in neuronal activity in CGp specifically, and the default network more generally, are necessary for cognitive control (Gusnard and Raichle, 2001; Raichle et al., 2001; Raichle and Mintun, 2006; Mason et al., 2007; Buckner et al., 2008; Hayden et al., 2009).

Posterior cingulate cortex is situated at neuroanatomical crossroads, linking the anterior cingulate cortex (ACC) and other limbic structures, the parietal cortex, and the hippocampus. Lesion and neuroimaging evidence indicates that ACC plays a critical role in task switching (Sohn et al., 2000; Rushworth et al., 2002, 2003; Brass et al., 2005; Crone et al., 2006; Johnston et al., 2007). A combined fMRI/TMS study demonstrated that ACC and posterior supplementary motor area (pSMA) are activated following task switches, and that disrupting pSMA with transcranial magnetic stimulation (TMS) increases delays associated with switching (Rushworth et al., 2002). Moreover, lesions in ACC impair task-switching in monkeys without affecting delayed alternation behavior (i.e., alternating between two food wells without switching tasks, Rushworth et al., 2003).

Supporting these imaging and lesion data, ACC neurons exhibit a strong increase in task-specific preparatory firing following task switches that gradually diminishes on subsequent trials; this effect is not observed in the dorsolateral prefrontal cortex (DLPFC, Johnston et al., 2007). Complementary studies show that neurons in SMA increase spiking following switches (Isoda and Hikosaka, 2007) and alter their firing as a function of task preparation and task set (Hoshi and Tanji, 2004). Neuroimaging studies of humans and monkeys have implicated dorsal lateral prefrontal cortex in task switching as well (Dove et al., 2000; Nakahara et al., 2002; Aron et al., 2004).

\section{REFERENCES}

Altmann, E. M. (2004). Advance preparation in task switching: what work is being done? Psychol. Sci. 15, 616-622.

Aron, A. R., Robbins, T. W., and Poldrack, R. A. (2004). Inhibition and the right inferior frontal cortex. Trends Cogn. Sci. 8, 170-177.

Boly, M., Balteau, E., Schnakers, C., Degueldre, C., Moonen, G., Luxen, A., Phillips, C., Peigneux, P., Maquet, P., and Laureys, S. (2007). Baseline brain activity fluctuations predict somatosensory perception in humans. Proc. Natl. Acad. Sci. U.S.A. 104, 12187-12192.

Brainard, D. H. (1997). The psychophysics toolbox. Spat. Vis. 10, 433-436.

Brass, M., Ullsperger, M., Knoesche, T. R., von Cramon, D. Y., and Phillips, N. A. (2005). Who comes first? The role of the prefrontal and parietal cortex in cognitive control. J. Cogn. Neurosci. $17,1367-1375$.

Finally, single neurons in the parietal lobe encode abstract variables related to task set (Stoet and Snyder, 2004). One possibility is that CGp functionally links frontomedial structures on one hand and parietal structures on the other to effect cognitive control.

Posterior cingulate cortex is distinguished from these other areas by showing reduced neuronal activity following task switches, with gradually increasing firing rates on later trials. This inverse correlation is consistent with the idea that this area mediates cognitive processes that compete with or are silenced by processes that recruit exteroceptive vigilance (Gusnard et al., 2001; Mason et al., 2007; Hayden et al., 2009). Such processes may include forms of attention and arousal, environmental monitoring, memory consolidation, self-referential thought, or even mind wandering (Gusnard et al., 2001; Daselaar et al., 2004; Raichle and Gusnard, 2005; Boly et al., 2007; Mason et al., 2007; Buckner et al., 2008). Whereas the majority of neurophysiological studies have focused on brief, high-amplitude neuronal events, CGp is distinguished by its reliably low amplitude, long-lasting changes in firing rates (Hayden et al., 2008). We have previously suggested that these slow, weak variations in activity may efficiently track important state variables that persist across many trials (Hayden et al., 2008, 2009).

We acknowledge that the present data do not resolve the major controversies surrounding the provenance of switching costs. As noted, switching costs may reflect cognitive control processes associated with task set reconfiguration (Monsell, 2003), memory limitations (Wylie and Allport, 2000; Altmann, 2004), perceptual encoding of the cue (Logan and Bundesen, 2003), priming (Schneider and Logan, 2005; Logan et al., 2007), or activating a new task set (Altmann, 2004). Indeed, the present results, like other taskswitching results, are consistent with the idea that neuronal activity in CGp closely tracks vigilance, arousal, or attention - processes that in turn are influenced in a systematic manner by switching between tasks. Nonetheless, the present results implicate CGp, and by extension, the brain's default network - the poorly understood "dark energy" of the brain (Raichle, 2006) - in the cognitive processes that mediate disengagement from one task to perform another.

\section{ACKNOWLEDGMENTS}

We thank Vinod Venkatraman for useful comments in the design of the experiment and the analysis. This work was supported by the National Eye Institute of the National Institutes of Health (grant number EY013496 to Michael L. Platt), by a Fellowship from the Tourette Syndrome Association, and by a K-99 award from the National Institute of Drug Abuse (027718-01) to Benjamin Y. Hayden.

Buckner, R. L., Andrews-Hanna, J. R. and Schacter, D. L. (2008). The brain's default network: anatomy, function, and relevance to disease. Ann. N. Y. Acad. Sci. 1124, 1-38.

Corbetta, M., Kincade, J. M., and Shulman, G. L. (2002). Neural systems for visual orienting and their relationships to spatial working memory. J. Cogn. Neurosci. 14, 508-523.

Cornelissen, F. W., Peters, E., and Palmer, J. (2002). The eyelink toolbox: eye tracking with MATLAB and the psychophysics toolbox. Behav. Res. Methods Instrum. Comput. 34, 613-617.

Crone, E. A., Wendelken, C., Donohue, S. E., and Bunge, S. A. (2006). Neural evidence for dissociable components of task-switching. Cereb. Cortex 16, 475-486.

Daselaar, S. M., Prince, S. E., and Cabeza, R. (2004). When less means more: deactivations during encoding that predict subsequent memory. Neuroimage 23, 921-927. 
Dean, H. L., Crowley, J. C., and Platt, M. L. (2004). Visual and saccaderelated activity in macaque posterior cingulate cortex. J. Neurophysiol. 92, 3056-3068.

Dove, A., Pollmann, S., Schubert, T., Wiggins, C. J., and von Cramon, D. Y. (2000). Prefrontal cortex activation in task switching: an event-related fMRI study. Brain Res. Cogn. Brain Res. 9, 103-109.

Fox, M. D., Snyder, A. Z., Vincent, J. L., Corbetta, M., Van Essen, D. C., and Raichle, M. E. (2005). The human brain is intrinsically organized into dynamic, anticorrelated functional networks. Proc. Natl. Acad. Sci. U.S.A. 102, 9673-9678.

Glimcher, P.W., Ciaramitaro, V. M., Platt, M. L., Bayer, H. M., Brown, M. A., and Handel, A. (2001). Application of neurosonography to experimental physiology. J. Neurosci. Methods 108, 131-144.

Gusnard, D. A., Akbudak, E., Shulman, G. L., and Raichle, M. E. (2001). Medial prefrontal cortex and self-referential mental activity: relation to a default mode of brain function. Proc. Natl. Acad. Sci. U.S.A. 98, 4259-4264.

Gusnard, D. A., and Raichle, M.E. (2001). Searching for a baseline: functional imaging and the resting human brain. Nat. Rev. Neurosci. 2, 685-694.

Hayden, B. Y., Nair, A. C., McCoy, A. N., and Platt, M. L. (2008). Posterior cingulate cortex mediates outcomecontingent allocation of behavior. Neuron 60, 19-25.

Hayden, B. Y., Smith, D. V., and Platt, M. L. (2009). Electrophysiological correlates of default-mode processing in macaque posterior cingulate cortex. Proc. Natl. Acad. Sci. U.S.A. 106, 5948-5953.

Hoshi, E., and Tanji, J. (2004). Differential roles of neuronal activity in the supplementary and presupplementary motor areas: from information retrieval to motor planning and execution. J. Neurophysiol. 92, 3482-3499.

Hyafil, A., Summerfield, C., and Koechlin, E. (2009). Two mechanisms for task switching in the prefrontal cortex. J. Neurosci. 29, 5135-5142.

Isoda, M., and Hikosaka, O. (2007). Switching from automatic to controlled action by monkey medial frontal cortex. Nat. Neurosci. 10, 240-248.

Johnston, K., Levin, H. M., Koval, M. J., and Everling, S. (2007). Top-down control-signal dynamics in anterior cingulate and prefrontal cortex neurons following task switching. Neuron 53, 453-462.

Kobayashi, Y., and Amaral, D. G. (2003). Macaque monkey retrosplenial cortex: II. Cortical afferents. J. Comp. Neurol. 466, 48-79.

Logan, G. D., and Bundesen, C. (2003). Clever homunculus: is there an endogenous act of control in the explicit task-cuing procedure? J. Exp. Psychol. Hum. Percept. Perform. 29, 575-599.

Logan, G. D., Schneider, D. W., and Bundesen, C. (2007). Still clever after all these years: searching for the homunculus in explicitly cued task switching. J. Exp. Psychol. Hum. Percept. Perform. 33, 978-994.

Mason, M. F., Norton, M. I., Van Horn, J. D., Wegner, D. M., Grafton, S. T., and Macrae, C. N. (2007). Wandering minds: the default network and stimulus-independent thought. Science 315, 393-395.

McCoy, A. N., Crowley, J. C., Haghighian, G., Dean, H. L., and Platt, M. L. (2003). Saccade reward signals in posterior cingulate cortex. Neuron 40, 1031-1040.

McCoy, A. N., and Platt, M. L. (2005). Risk-sensitive neurons in macaque posterior cingulate cortex. Nat. Neurosci. 8, 1220-1227.

McKiernan, K. A., Kaifman, J. N., and Kucera-Thompson, J, Binder, J. R. (2003). A parametric manipulation of factors affecting task-induced deactivation in functional neuroimaging. J. Cogn. Neurosci. 15, 394-408.

Monsell, S. (2003). Task switching. Trends Cogn. Sci. 7, 134-140.

Nakahara, K., Hayashi, T., Konishi, S., and Miyashita, Y. (2002). Functional
MRI of macaque monkeys performing a cognitive set-shifting task. Science 295, 1532-1536.

Raichle, M. E. (2006). Neuroscience. The brain's dark energy. Science 314, 1249-1250.

Raichle, M.E., and Gusnard, D.A. (2005). Intrinsic brain activity sets the stage for expression of motivated behavior. J. Comp. Neurol. 493, 167-176.

Raichle, M. E., MacLeod, A. M., Snyder, A. Z., Powers, W. J., Gusnard, D. A., and Shulman, G. L. (2001). A default mode of brain function. Proc. Natl. Acad. Sci. U.S.A. 98, 676-682.

Raichle, M. E., and Mintun, M. A. (2006). Brain work and brain imaging. Annu Rev. Neurosci. 29, 449-476.

Rogers, R. D., and Monsell, S. (1995) Costs of a predictable switch between simple cognitive tasks. J. Exp. Psychol. Gen. 123, 207-231.

Rushworth, M. F., Hadland, K.A., Gaffan, D., and Passingham, R. E. (2003). The effect of cingulate cortex lesions on task switching and working memory. J. Cogn. Neurosci. 15, 338-353.

Rushworth, M. F., Hadland, K. A., Paus, T., and Sipila, P. K. (2002). Role of the human medial frontal cortex in task switching: a combined fMRI and TMS study. J. Neurophysiol. 87, 2577-2592.

Schneider, D. W., and Logan, G. D. (2005). Modeling task switching without switching tasks: a short-term priming account of explicitly cued performance. J. Exp. Psychol. Gen. 134, 343-367.

Smith, D.V., Davis, B., Niu, K., Healy, E. W., Bonilha, L., Fridriksson, J., Morgan, P., and Rorden, C. (2010). Spatial attention evokes similar activation patterns for visual and auditory stimuli.J. Cogn. Neurosci. 22, 347-361.

Sohn, M. H., Ursu, S., Anderson, J. R., Stenger, V.A., and Carter, C. S. (2000). Inaugural article: the role of prefrontal cortex and posterior parietal cortex in task switching. Proc. Natl. Acad. Sci. U. S. A. 97, 13448-13453.

Stoet, G., and Snyder, L. H. (2004). Single neurons in posterior parietal cortex of monkeys encode cognitive set. Neuron 42, 1003-1012.
Stoet, G., and Snyder, L. H. (2007). Extensive practice does not eliminate human switch costs. Cogn. Affect. Behav. Neurosci. 7, 192-197.

Stoet, G., and Snyder, L. H. (2009). Neural correlates of executive control functions in the monkey. Trends Cogn. Sci. 13, 228-234.

Vincent, J. L., Patel, G. H., Fox, M. D., Snyder, A. Z., Baker, J. T., Van Essen, D. C., Zempel, J. M., Snyder, L. H., Corbetta, M., and Raichle, M. E. (2007). Intrinsic functional architecture in the anaesthetized monkey brain. Nature 447, U83-U84.

Vincent, J. L., Snyder, A. Z., Fox, M. D., Shannon, B. J.,Andrews, J.R., Raichle, M. E., and Buckner, R. L. (2006). Coherent spontaneous activity identifies a hippocampal-parietal memory network. J. Neurophysiol. 96, 3517-3531.

Weissman, D. H., Roberts, K. C., Visscher, K. M., and Woldorff, M. G. (2006). The neural bases of momentary lapses in attention. Nat. Neurosci. 9, 971-978.

Wylie, G., and Allport, A. (2000). Task switching and the measurement of “switch costs." Psychol. Res. 63, 212-233.

Conflict of Interest Statement: The authors declare that the research was conducted in the absence of any commercial or financial relationships that could be construed as a potential conflict of interest.

Received: 24 September 2010; accepted: 18 November 2010; published online: 06 December 2010.

Citation: Hayden BY, Smith DV and Platt ML (2010) Cognitive control signals in posterior cingulate cortex. Front. Hum. Neurosci. 4:223. doi: 10.3389/ fnhum.2010.00223

Copyright (c) 2010 Hayden, Smith and Platt. This is an open-access article subject to an exclusive license agreement between the authors and the Frontiers Research Foundation, which permits unrestricted use, distribution, and reproduction in any medium, provided the original authors and source are credited. 